\title{
Urethral obstruction from Cobb's collar in association with VACTERL
}

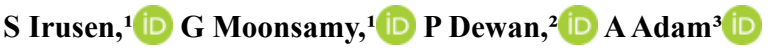 \\ ${ }^{1}$ Division of Paediatric Nephrology, Department of Paediatrics and Child Health, School of Clinical Medicine, Faculty of \\ Health Sciences, University of the Witwatersrand, South Africa \\ ${ }^{2}$ Kind Cuts for Kids, Australia \\ ${ }^{3}$ Division of Urology, University of the Witwatersrand, South Africa; Department of Urology, Charlotte Maxeke Johannesburg Academic \\ and Helen Joseph and Rahima Moosa Mother and Child (Coronation) Cluster Hospitals, South Africa; and Wits Donald Gordon Medical \\ Centre, South Africa
}

Corresponding author, email: aadam81@gmail.com

\begin{abstract}
Summary
Cobb's collar is a rare intraluminal congenital cause of bulbar urethral obstruction that is commonly mislabelled congenital obstructive posterior urethral membrane (COPUM) but may be distinguished from this endoscopically as it has no connecting folds to the verumontanum. In this report, Cobb's collar is described for the first time in association with the VACTERL syndrome. The abnormality was diagnosed and treated by diathermy and dilatation at the time of endoscopic evaluation, with improvement of vesicoureteric reflux on micturating cystourethrogram and renal ultrasound. Keywords: Cobb's collar, VACTERL association, posterior urethral valves, Moormann's ring, COPUM
\end{abstract}

Supplementary video available online: http://sajs.redbricklibrary.com/index.php/sajs/article/view/3527

\section{Case presentation}

A one-year-old male patient presented with a history of repeated episodes of bladder outlet obstruction and urinary retention with difficulty passing a transurethral catheter. On examination, his bladder was palpated inferior to the umbilicus. A 5 French feeding tube was passed via the urethra to decompress the bladder, but this was unsuccessful. Urgent ultrasound showed a full bladder with bilateral hydrouretero-nephrosis and progressive parenchymal effacement. He was referred to the paediatric urological services for further management.

In the neonatal period, the patient was diagnosed with VACTERL (vertebral defects, anal atresia, cardiac abnormalities, trachea-oesophageal fistula, renal anomalies and limb abnormalities) association. A dividing colostomy was performed on day 2 of life for an imperforate anus, and a haemodynamically insignificant patent ductus arteriosus was noted. Based on the renal and bladder ultrasound and micturating cystourethrogram (MCUG) (Figure 1) that showed a dilated posterior urethra, a diagnosis of a congenital obstructive posterior urethral membrane (COPUM) was considered most likely. However, cystoscopic evaluation (Supplementary Video 1) revealed urethral narrowing distal to the verumontanum (Figure $2 \mathrm{a}$ ), which required resection with diathermy and dilation.

The presence of a Cobb's collar in the bulbar urethra was diagnosed, previously mislabelled as a posterior urethra obstruction; his bladder neck and prostatic urethra were noted to be endoscopically normal with direct visualisation. No folds were leading from the obstructing lesion on direct visualisation (Figure $2 b$ ). Following surgery, the patient had fewer confirmed urinary tract infections (UTIs) and showed improvement in vesicoureteric reflux (VUR) on MCUG and renal ultrasound but did require further urethral dilation. This is the first reported case of a fibrous Cobb's collar described in conjunction with VACTERL association.

\section{Discussion}

Obstruction to the posterior urethra was first reported in 1717 by Morgagni and again in 1802 by Langenbeck. ${ }^{1}$ Subsequently, Young's seminal paper reviewed endoscopic and autopsy findings in 12 patients diagnosed with posterior urethral obstruction conceiving three variations of membranous obstruction with a six part classification. ${ }^{2}$ Cobb's collar was first identified in 1967 (or Moormann's ring - 1972) to describe an apparent congenital muscular or fibrous obstruction of the proximal bulb of the urethra distal to the external bladder sphincter and distinct from the verumontanum distal to the membranous urethra. ${ }^{3}$ If fibrous, it was hypothesised to result embryologically from incomplete rupture of the cloacal membrane..$^{1}$ The nomenclature of previously understood and accepted classification terms of urethral blockage underwent further revision. The unified morphology of COPUM (Young's type I and II valve that have attachments to the verumontanum) for posterior urethral obstruction, and Cobb's collar (Young's type III valve) if found in the bulbar urethra was to replace Young's original classification. ${ }^{4}$

The precise incidence of Cobb's collar is presently unknown as patients are often mislabelled with a high level of obstruction. Presentation, however, is bimodal with the diagnosis being made in infancy and the second decade 

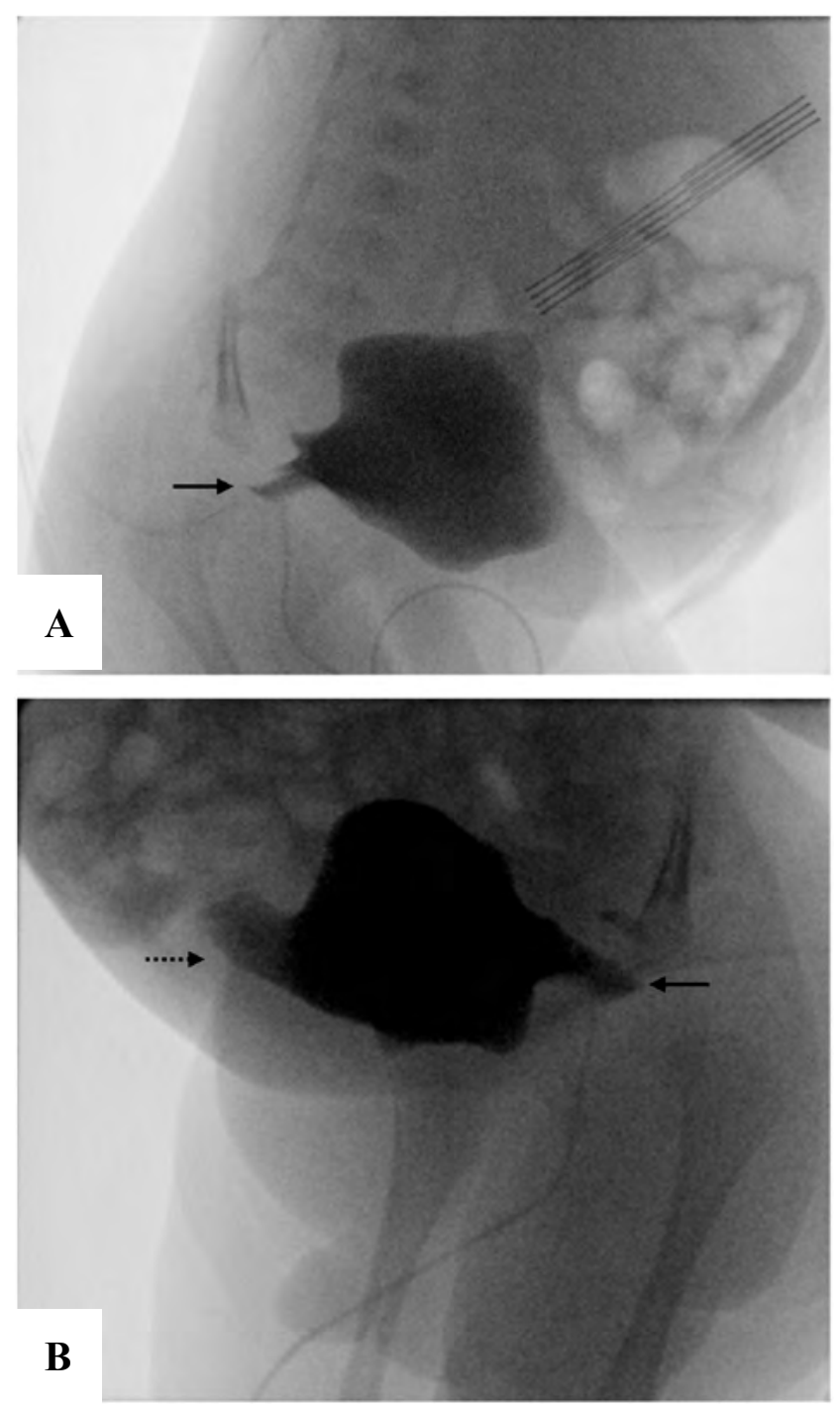

Figure 1: Micturating cystourethrogram showing abnormally shaped bladder with dilated posterior urethra (A; solid arrow) and urachal diverticulum $(B$; dashed arrow)

of life when patients present with febrile UTIs, enuresis, urinary incontinence or acute retention. ${ }^{5}$

Patients who present younger than 6 months are more likely to have radiological evidence of bladder and upper renal tract changes on radiological imaging. MCUG findings may demonstrate narrowing of the bulbar urethra. This, however, may not directly correlate with the degree of obstruction seen on direct endoscope evaluation. ${ }^{6}$

It has been shown that patients can be successfully treated with cold knife incision or electrical cautery. The latter is associated with submucosal thermal injury and the development of de novo urethral strictures. ${ }^{5}$ Intervention has shown improvement in bladder irritability, VUR, urethral calibre and uroflowmetry as well as a reduced presentation with UTI and enuresis.., 7

This is the first reported case of a Cobb's collar in association with VACTERL. VACTERL was first described in 1973 and is defined by the presence of at least three of the described abnormalities, with anorectal malformations and trachea-oesophageal fistulae considered core components. ${ }^{8}$ The estimated incidence is between 1 in 10000 and 1 in 40000 live births. ${ }^{8}$ The underlying aetiology is identified in only a small proportion of newborn babies as there is a high
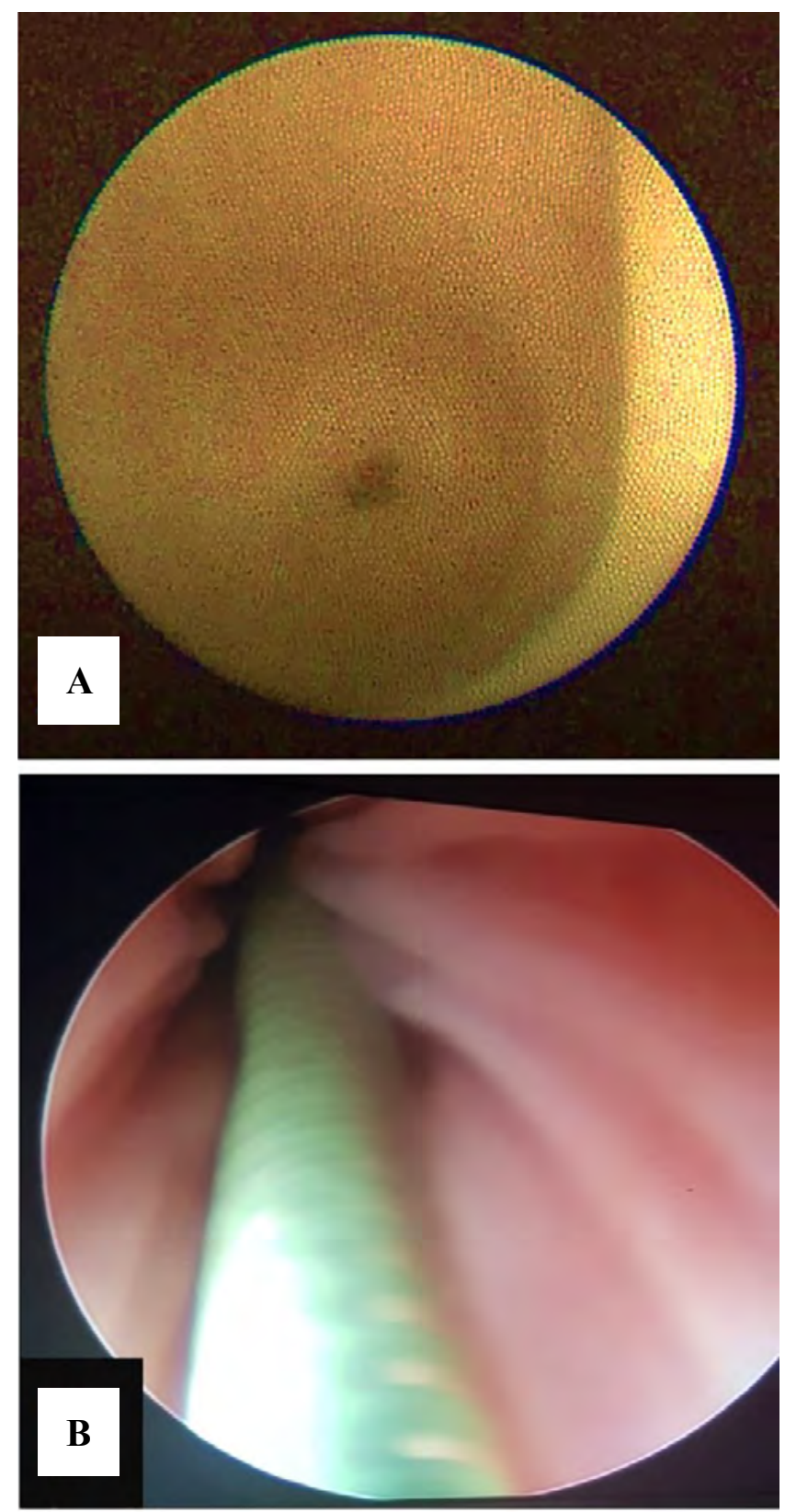

Figure 2: Endoscopic view of Cobb's collar (A) and normal prostatic urethra distal to identified urethral obstruction (B)

degree of clinical and causal heterogenicity. Hypotheses include primary polytopic developmental field defect with familial clustering and mutations in sonic hedgehog pathway genes as shown in animal models. ${ }^{8}$

Common renal anomalies found in patients with VACTERL association include VUR, renal agenesis (unior bilateral), dysplastic or multicystic kidneys and duplex kidney collecting systems. ${ }^{9}$ One case of lower urinary tract obstruction secondary to urethral stenosis in a patient with VACTERL has been described; the patient was treated with intrauterine foetal vesicoamniotic shunting successfully. ${ }^{10}$

\section{Conclusion}

Cobb's collar is a rare cause of posterior bulbar urethral obstruction. At the time of endoscopic evaluation, it can be diagnosed as it has no connecting folds to the verumontanum and treated by incision and dilatation with expected improvement in VUR and bladder uroflowmetry. 
This is the first description of association of Cobb's collar with the VACTERL syndrome, though the relationship may be incidental.

\section{Conflict of interest}

The authors declare no conflict of interest.

\section{Funding source}

No funding was required.

\section{Ethical approval}

Signed informed individual consent was obtained from the patient's mother. Local institutional ethical approval was attained, with Human Research Ethics Committee (Medical) Clearance certificate - M200770 (2020/07/08).

\section{ORCID}

S Irusen (iD) https://orcid.org/0000-0001-6205-3181

G Moonsamy (iD https://orcid.org/0000-0001-6453-0252

P Dewan (iD) https://orcid.org/0000-0003-2808-7921

A Adam iD https://orcid.org/0000-0001-9069-3282

\section{REFERENCES}

1. Dewan PA. Posterior urethral obstruction. Bangladesh J Endosurgery. 2014;2:29-32. https://doi.org/10.1007/BF02353 001

2. Young H, Frontz W, Baldwin J. Congenital obstruction of the posterior urethra. J Urol. 1919;3:289-365.
3. Cobb BG, Wolf JA, Ansell JS. Congenital stricture of the proximal urethral bulb. J Urol. 1968;99(5):629-31. https://doi. org/10.1016/S0022-5347(17)62761-7.

4. Dewan PA. Congenital obstructing posterior urethral membranes (COPUM) - further evidence for a common morphological diagnosis. Pediatr Surg Int. 1993;8:45-50. https://doi.org/10.1007/BF02353001.

5. Nonomura K, Kanno T, Kakizaki H, et al. Impact of congenital narrowing of the bulbar urethra (Cobb's collar) and its transurethral incision in children. Eur Urol. 1999;36(2):1449. https://doi.org/10.1159/000067986.

6. Lu Y, Dewan PA. Congenital urethral obstruction - the videoendoscopic perspective. BJU Int. 2006;98(5):953-9. https:/ doi.org/10.1111/j.1464-410X.2006.06420.x.

7. Adorisio O, Bassani F, Silveri M. Cobb's collar - a rare cause of urinary retention. BMJ Case Rep. 2013;2013:2012-4. https://doi.org/10.1136/bcr-2012-008137.

8. Solomon BD. The etiology of VACTERL association current knowledge and hypotheses. Am J Med Genet. 2018;178(4):440-6. https://doi.org/10.1002/ajmg.c.31664.

9. Cunningham BK, Khromykh A, Martinez AF, et al. Analysis of renal anomalies in VACTERL association. Birth Defects Res Part A - Clin Mol Teratol. 2014;100(10):801-5. https:// doi.org/10.1002/bdra.23302.

10. Kanasugi T, Kikuchi A, Haba G, et al. Vesico-amniotic shunting for lower urinary tract obstruction in a fetus with VACTERL association. Congenit Anom (Kyoto). 2016;56(5):237-9. https://doi.org/10.1111/cga.12166. 